\title{
LAS SIMULACIONES EN ENTORNOS TIC COMO HERRAMIENTA PARA LA FORMACIÓN EN COMPETENCIAS TRANSVERSALES DE LOS ESTUDIANTES UNIVERSITARIOS
}

\section{Resumen:}

Este artículo ofrece una reflexión sobre cómo los entornos tecnológicos pueden jugar un papel fundamental en el actual escenario de la educación superior considerando tanto su configuración estructural como los agentes clave del proceso educativo.

El contenido del texto se desarrolla primeramente contextualizando el estudiante en la Universidad del siglo XXI; se analiza la renovación metodológica desde una óptica del desarrollo de las tecnologías y ofreciendo una visión del rol de profesor y estudiante en este nuevo escenario; finalmente se proponen las simulaciones en entornos tecnológicos como una estrategia formativa muy valiosa para dar respuesta a las necesidades formativas del estudiante en la sociedad actual.

Palabras clave:

Educación Superior, Tecnologías de la Información y las Comunicaciones, competencias Transversales, simulación.

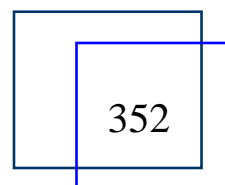

Mercè Gisbert Cervera, Jose M. Cela-Ranilla y Sofia Isus Barado 


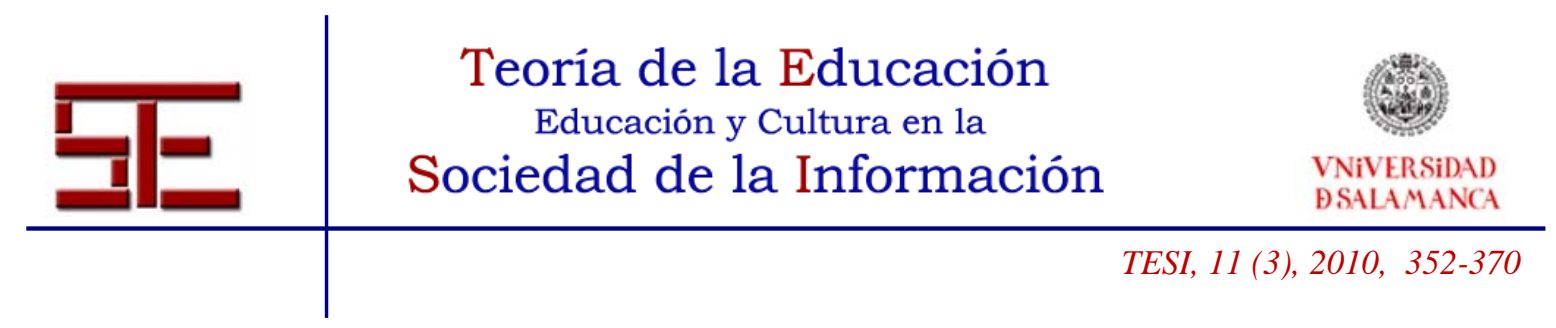

SIMULATIONS IN TECHNOLOGICAL ENVIRONMENTS AS A TOOL FOR TRAINING IN TRANSVERSAL COMPETENCES FOR UNIVERSITY STUDENTS

\begin{abstract}
:
This paper consists of a reflection on how the technological environments can play a key role in the current Higher Education scene. This reflection observes the structural configuration and the key agents of the educational process. The content is developed firstly locating the student in the University of the 21st century; the methodological renovation is analyzed from two perspectives: the development of the technologies and the new role of teacher and student in this new scene; finally the simulations in technological environments are proposed as a valuable strategy to give response to the formative needs of the student in the current society.
\end{abstract}

Key words:

Higher Education, Information and Communication Technologies, Transversal Competences, simulations.

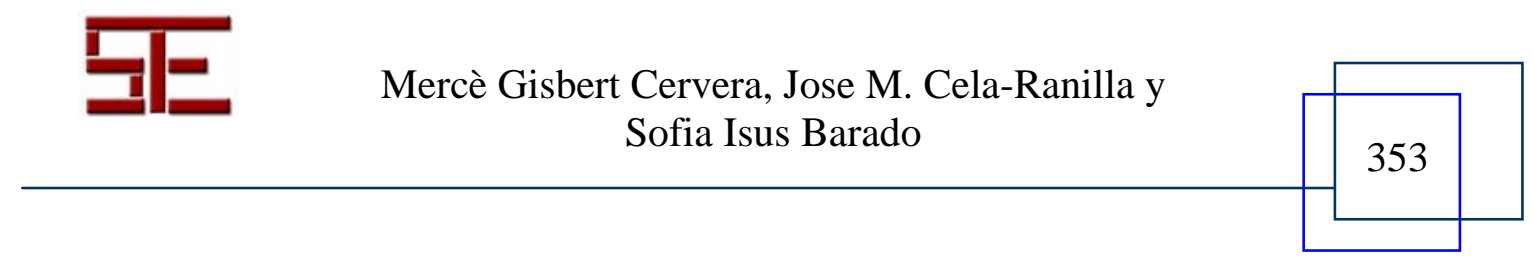




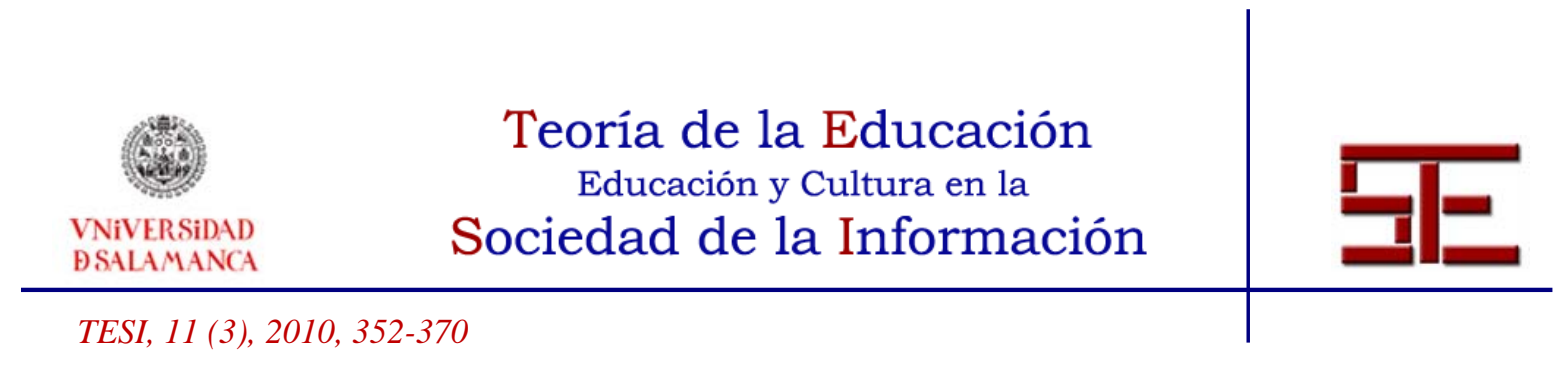

\title{
LAS SIMULACIONES EN ENTORNOS TIC COMO HERRAMIENTA PARA LA FORMACIÓN EN COMPETENCIAS TRANSVERSALES DE LOS ESTUDIANTES UNIVERSITARIOS
}

Fecha de recepción: 02/01/2010; fecha de aceptación: 02/02/2010; fecha de publicación: 28/02/10

\author{
Mercè Gisbert Cervera \\ merce.gisbert@urv.cat \\ Universidad Rovira i Virgili. \\ Jose M. Cela-Ranilla \\ josemaria.cela@urv.cat \\ Universidad Rovira i Virgili \\ Sofia Isus Barado \\ sisus@pip.udl.cat \\ Universidad de Lleida.
}

\section{1.- INTRODUCCIÓN}

La sociedad actual ha establecido unas normas donde el potencial humano determina los cambios en todo su sistema de relaciones. Este potencial humano ha de verse estimulado por unos sistemas de formación al servicio de lo que las personas necesitan para desarrollarse personalmente y para dar respuesta a los requerimientos que la propia sociedad demanda.

La Universidad es un elemento clave dentro del proceso formativo de las personas. Por tanto, su trabajo debe abarcar desde la identificación de los requerimientos formativos de la sociedad y de las personas hasta propiciar los mecanismos más adecuados para llevar a cabo su acción educativa.

Su búsqueda de procesos educativos en términos de eficiencia y calidad debe estar atenta a la oportunidad que proporciona el desarrollo de las Tecnologías de la Información y las Comunicaciones (TIC). Estas tecnologías pueden ser observadas desde su versión más simple hasta la más compleja, sin embargo, su complejidad no determina su valor educativo sino el impacto que tenga ésta en la persona que aprende.

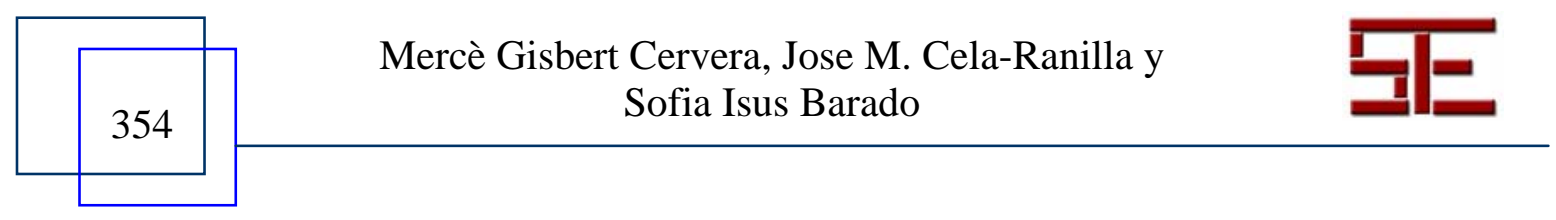




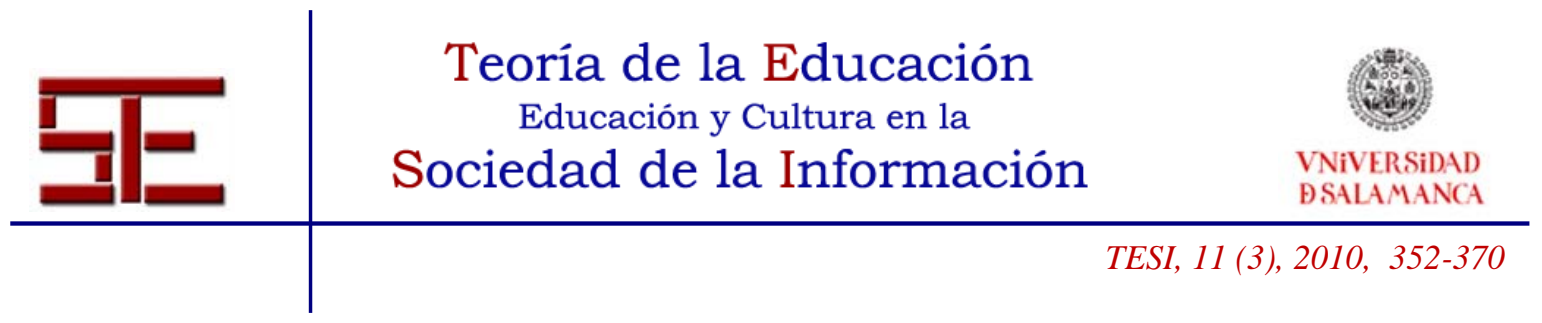

No obstante, no debe obviarse el potencial que ofrecen los avances tecnológicos; más bien al contrario, deben explorarse en toda su complejidad con una intencionalidad educativa. En este sentido, las simulaciones en entornos tecnológicos pueden constituir una estrategia capaz de generar situaciones de aprendizaje que den respuesta a las necesidades formativas en términos personales e institucionales.

Este artículo hace una reflexión sobre cómo los entornos tecnológicos, proponiendo concretamente la simulación, pueden jugar un papel fundamental en el actual escenario de la educación superior considerando tanto su configuración estructural como los agentes clave del proceso educativo.

\section{2.- UNIVERSIDAD Y ESTUDIANTE EN EL SIGLO XXI}

La década de este siglo XXI constituye una fecha clave en el futuro de la educación en el ámbito europeo. La consolidación de un espacio común en educación superior obliga a una toma de decisiones de las que dependerá el tipo de formación que se dispensará a los futuros ciudadanos europeos.

Las estructuras universitarias se organizan en torno a la formación de titulados en términos de calidad y en coherencia con una idea de ciudadanía en términos de bienestar y compromiso social. Por tanto, parece relevante describir brevemente en qué consiste esta coyuntura histórica así como analizar el tipo de universitario actual con la mirada en los futuros ciudadanos.

\subsection{Coyuntura europea: una oportunidad para cambiar}

La construcción de un EEES significa dotarse de un sistema de estructuras formativas compatibles, que permita y promueva el acceso a un mercado laboral con una dimensión europea. Este hecho se pone de manifiesto desde el diseño de los currícula formativos en términos de competencias hasta la puesta en marcha de organismos y mecanismos que permitan reconocer y garantizar la calidad de estos sistemas y su posterior desarrollo desde una perspectiva supranacional.

La esencia del proceso de armonización europea también se define por un cambio de paradigma educativo orientado a la centralidad de un alumno que necesita aprender durante toda la vida. Se trata de un modelo educativo donde el peso específico del proceso se desplaza del profesor y la enseñanza hacia el alumno y su aprendizaje.

Preparar a la Universidad de hoy para el aprendizaje del mañana significa la creación de un conocimiento dinámico y el uso de herramientas tecnológicas para el acceso a la

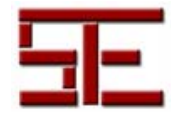

Mercè Gisbert Cervera, Jose M. Cela-Ranilla y Sofia Isus Barado 


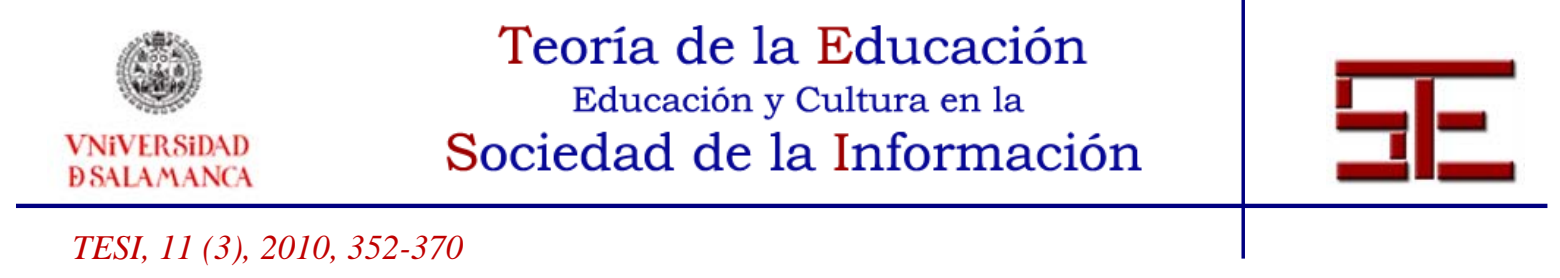

información y al conocimiento (Hartman et al., 2005). Esta realidad supone afrontar los desafíos económicos, sociales y educativos derivados de la inclusión de las TIC en la educación superior (Tondeur et al, 2007). Todo ello implica usar las herramientas tecnológicas personales como elementos clave de acceso a la información y al conocimiento y, por extensión, a los procesos de aprendizaje.

Una apuesta en esta dirección implica recursos, espacios y materiales adaptables y adaptados a las necesidades de los estudiantes, en definitiva, la personalización de servicios y de procesos de formación.

Los cambios en la educación superior relacionados con las TIC’s tienen que ver con:

- Las inversiones.

- Las herramientas TIC para la formación por competencias.

- Los espacios y procesos de formación interactivos.

- Usar las TIC como herramientas para mejorar la formación práctica.

- Usar las TIC para generar espacios reales en contextos virtuales.

Revisando el panorama internacional relacionado con las tendencias actuales en educación superior se evidencian una serie de líneas de investigación que teniendo al estudiante como referencia intentan definir, desarrollar e implementar nuevos entornos de formación considerando que las TIC’s serán un elemento clave.

El desarrollo tecnológico de los últimos años ha posibilitado que nuevas estrategias de aprendizaje, como el mLearning, ofrezcan valor añadido a las metodologías tradicionales dada su potencialidad para crear nuevas oportunidades de aprendizaje (White, 2007).

El mundo cotidiano del estudiante universitario es un mundo tecnológico, por tanto utilizar tecnología para favorecer su proceso de aprendizaje será una estrategia imprescindible para lograr la adaptación a las características de los estudiantes.

\subsection{El estudiante universitario: de estudiante profesional a profesional competente}

\section{Formación en base a competencias}

La necesidad de acercar la formación universitaria al mundo laboral y profesional implica cambiar también el contenido de los procesos de aprendizaje. El salto cualitativo que se hizo de las cualificaciones a las competencias en la década de los noventa en el mundo de la formación profesional debe hacerse ahora en la formación universitaria.

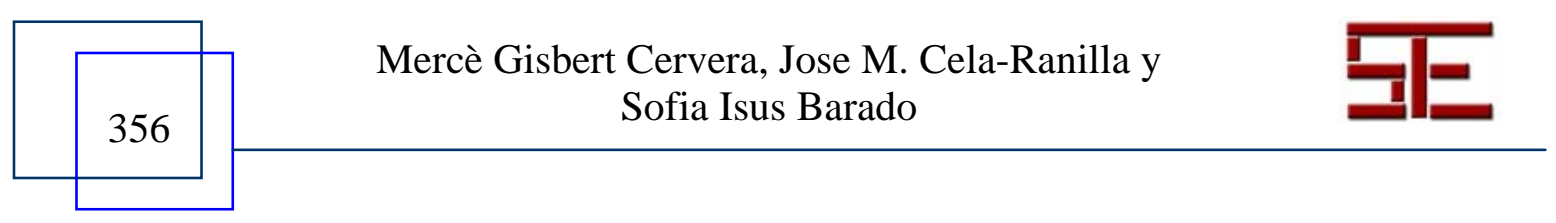




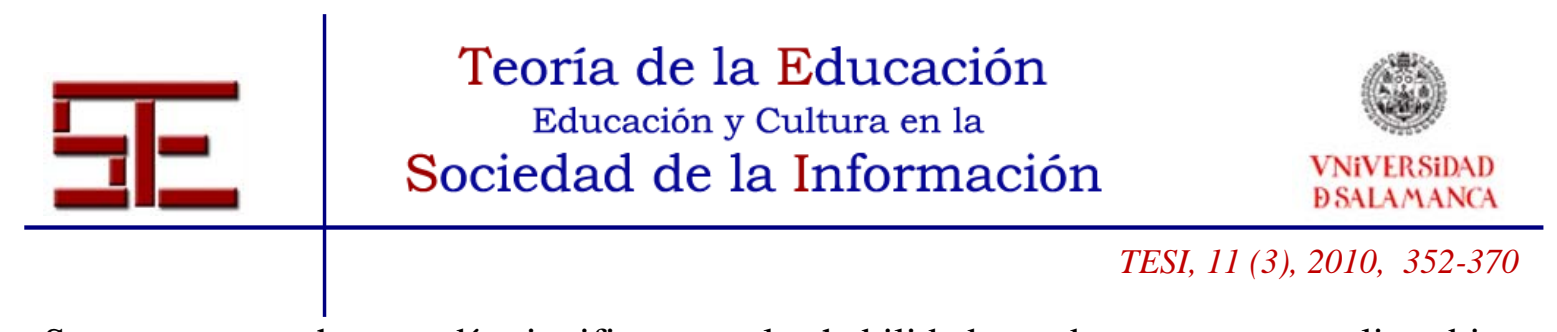

Ser competente hoy en día significa tener las habilidades y destrezas para realizar bien el trabajo, pero además saber resolver problemas con autonomía y colaborar en la organización del trabajo (Bunk,1994). Esta puesta en práctica de aptitudes, rasgos de personalidad, conocimientos adquiridos y también valores viene modelada por una serie de características que sin ser específicas de un determinado puesto de trabajo o de una determinada profesión, son necesarias para desempeñarse de forma competente en el nivel requerido por el empleo. Estas características son las denominadas competencias transversales, aquellas que además de ser transferibles a distintos contextos laborales, permiten y facilitan una continua adaptación a un mundo laboral que cambia continuamente en sus contenidos específicos.

\section{Aprendizaje de Competencias Transversales}

Una manera más amplia de definir las competencias transversales es entendiéndolas como un conjunto de habilidades de amplio alcance que afectan a distintas clases de tareas y que se desarrollan en situaciones distintas, por lo que son ampliamente generalizables y transferibles, dando como resultado una ejecución profesional eficaz.

En el campo universitario enseñar estas competencias transversales es posible en una acción conjunta que implique:

a) Incorporación de metodologías docentes que permitan desarrollar las competencias desde la programación de cada asignatura o módulo.

b) En las prácticas de empresa y entornos laborales.

c) A través de situaciones simuladas de entornos profesionales, que faciliten el transfer de conocimientos y actitudes en la práctica profesional.

Las competencias profesionales se aprenden en la acción. El nivel de desarrollo de las TIC en el campo educativo debería permitir realizar propuestas formativas en entornos tecnológicos que son similares a las realidades profesionales. Ofrecer competencias básicas en entornos tecnológicos es esencial para que los estudiantes puedan actuar eficazmente en el medio académico y laboral, así como en las actividades cotidianas (Ezziane, 2007).

Trabajar en entornos "reales" a través de herramientas tecnológicas de simulación permite tomar decisiones sobre situaciones en el mismo momento que se producen. Esto aporta un valor añadido a la formación superior ya que uno de las mayores críticas que recibe la universidad es su poca versatilidad y su poca capacidad de adaptarse a las necesidades del mundo en el que está inmersa para poder preparar de manera adecuada para el mundo laboral y profesional.

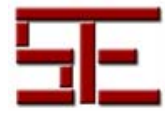

Mercè Gisbert Cervera, Jose M. Cela-Ranilla y Sofia Isus Barado 


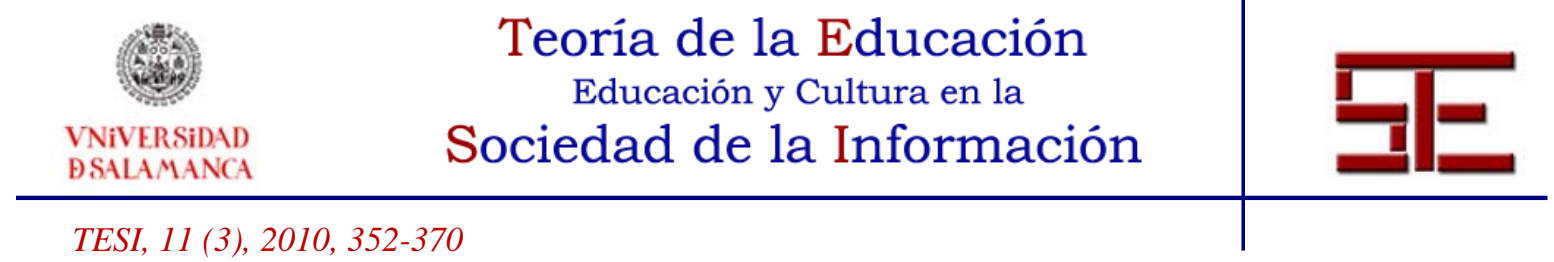

La mayor parte de las investigaciones que se han realizado sobre el proceso de aprendizaje por competencias enfatizan en que la eficiencia de éste tiene que ver con procesos de transfer, exploración activa y situaciones sociales. Esto implica que las situaciones que simulen entornos laborales pueden facilitar el proceso de aprendizaje por competencias.

\subsection{De las aulas a los entornos de aprendizaje}

Una de las misiones principales de la universidad es facilitar/favorecer procesos de aprendizaje eficientes en los estudiantes. Internet ha cambiado el espacio físico por el espacio virtual. El trabajo en el espacio virtual no sólo ha generado nuevas estrategias metodológicas sino también nuevos procesos cognitivos en los estudiantes pues los procesos y estilos de aprendizaje tienen otros referentes que no son sólo el profesor y los contenidos.

Un número considerable de estudios realizados en la última década apuntan a la necesidad de reformular el espacio de formación. Las aulas tradicionales se convierten, progresivamente, en espacios para la comunicación multimedia (Valenti, 2002; Fink, 2002; Nelson \& Soli, 2000; Roberts, 2005). El avance de la red y de la infraestructura de comunicaciones permite recrear, en situaciones de clase, toda la realidad del entorno local y global para que los alumnos puedan aprender a tomar decisiones y a adquirir competencias a partir de su interacción con la realidad del día a día desde dentro de los espacios universitarios. La tecnología, omnipresente en todos los ámbitos de la sociedad puede ser simple (ej. Mensajes de texto), aunque su impacto puede ser transformador (White, 2007).

Estudiar en espacios virtuales y con herramientas tecnológicas que simulan la realidad actual favorece el desarrollo de procesos cognitivos más complejos que pasan por la toma constante de decisiones a la vez que requieren de un grado de autogestión del proceso de aprendizaje por parte de los estudiantes.

Hablar de entornos de aprendizaje supone hablar de un ámbito y un proceso mucho más sofisticado que el aula en si. En la Universidad hemos de superar la noción de las cuatro paredes de las aulas para pasar a los verdaderos entornos para el aprendizaje que requieren otra concepción del espacio y del tiempo de formación. Un tiempo y un espacio flexible y adaptable a las necesidades y exigencias del estudiante (NLII, 2004; Scott-Webber, 2004; Mitchell, 2004). Un estudio reciente demuestra que los centros educativos más productivos son aquellos que más y mejor hacen uso de las TIC para mejorar sus procesos educativos, potenciando tanto la investigación como la innovación (Xu et al., 2007).

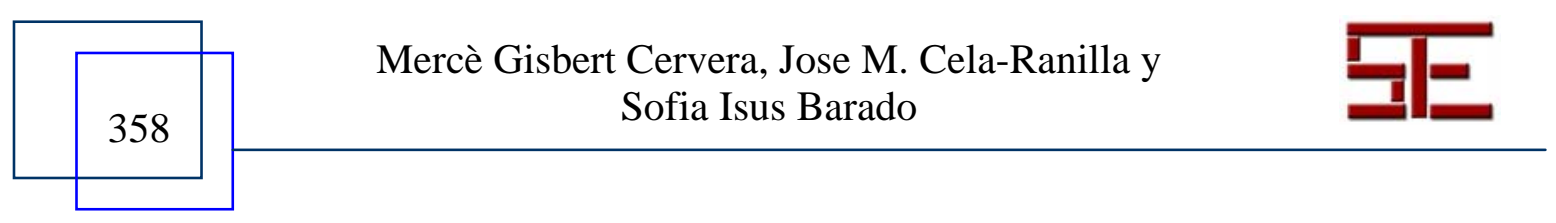




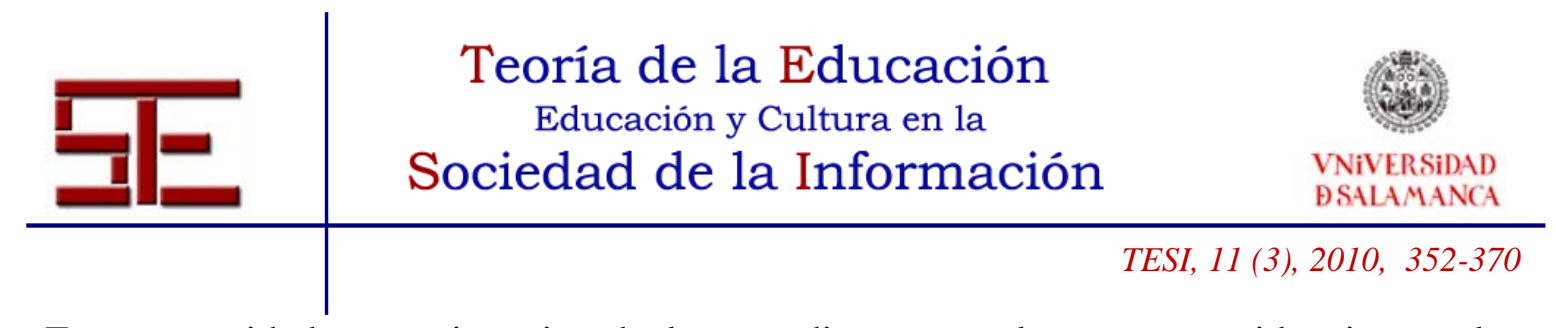

Estas necesidades y exigencias de los estudiantes son las que se evidencian en los principios que fundamentan el EEES en el que se considera al estudiante y a su proceso de aprendizaje el centro del proceso mismo de formación. En este sentido, la reforma de las universidades deberá plantearse los siguientes retos:

- $\quad$ El proceso de aprendizaje de los estudiantes. Aprendizaje activo:
o Trabajar juntos sobre problemas reales
o Interactuar con información y comunidades más allá del espacio del aula
o Debatir, investigar y resolver problemas
o Utilizar herramientas de simulación de juego de roles y de sensaciones y viajes virtuales

- La implementación de Campus Interactivos en los que las TIC’s tendrán un papel fundamental. Para definir y rediseñar de manera adecuada los campus y los espacios para el aprendizaje tendremos en cuenta:
o Las necesidades específicas de las disciplinas
o Las referencias externas y las experiencias de otras instituciones y colegas
o Los diversos estilos de aprendizaje de los estudiantes.
o Los espacios disponibles y las nuevas necesidades a partir de la implementación de nuevas herramientas.
o Las reformas curriculares (grados, postgrados, doctorados).
o Las actividades multidisciplinares y en contextos internacionales globales.

La sociedad en general y los sistemas educativos en particular deben asumir la responsabilidad de formar a los ciudadanos para permitirles utilizar las TIC y especialmente las redes telemáticas para su mejora personal y profesional y para favorecerles su acceso al conocimiento y los recursos multimedia. Esta sociedad debe asumir la responsabilidad de potenciar el uso y desarrollo de materiales y entornos tecnológicos de formación de calidad que permitan garantizar la competencia personal y profesional de sus usuarios así como su capacidad de autoaprendizaje y autoformación que les permitan, no sólo adaptarse a las transformaciones de una sociedad en cambio constante, sino también tomar parte activa y efectiva en las mismas.

Por otra parte, será necesario crear espacios de formación complementarios, paralelos e incluso alternativos para diferentes colectivos:

- Aquellos que fracasan reiteradamente en el sistema educativo formal y que, aún así, precisan seguir formándose.

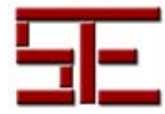

Mercè Gisbert Cervera, Jose M. Cela-Ranilla y Sofia Isus Barado 


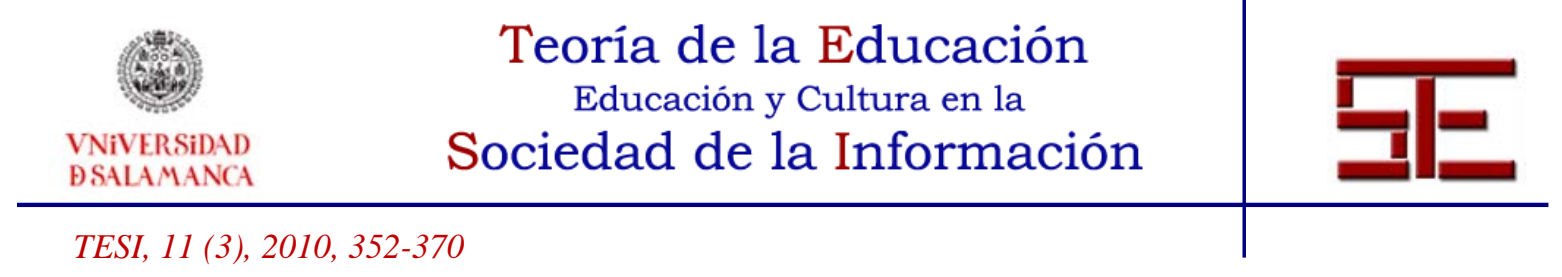

- La población activa de la que el mercado laboral exige cada vez más y en menos tiempo.

- Los jubilados, que también están ya utilizando las TIC como herramienta habitual para comunicarse, formarse y continuar aprendiendo.

\section{3.- RENOVACIÓN METODOLÓGICA Y TIC}

En coherencia con el nuevo modelo educativo, el nuevo perfil de alumno y los requerimientos profesionales en el entorno laboral, se hace necesaria una renovación y actualización de las metodologías docentes a utilizar en los procesos formativos.

La praxis docente debe tender a propuestas basadas en la diversidad de estrategias y en metodologías que impliquen actividad; en definitiva, proponer escenarios formativos donde el alumno pueda poner en práctica de forma integrada las competencias que necesita.

Esta filosofía de partida encuentra un gran aliado en las oportunidades que proporciona el desarrollo de la tecnología, en tanto permite crear entornos adaptados a múltiples perfiles de alumnos, con situaciones próximas a la práctica profesional, pudiendo interactuar de manera autónoma o en colaboración y con la posibilidad añadida de gestionar una supervisión y seguimiento para acompañar el proceso de forma adecuada. Recientes etudios señalan la contribución de las tecnologías basadas en juegos y simulaciones para superar dificultades en los procesos de aprendizaje (Connolly and Stansfield, 2007).

La trascendencia de este doble componente se hace explicita en la consecución de diferentes proyectos vinculados a las TIC (Michavila, 2004; Mora, 2005) o la formación de comisiones de trabajo impulsadas desde el ministerio competente (MEC, 2006).

Sin embargo, no toda actividad realizada a través de entornos tecnológicos conlleva un incremento del aprendizaje. De ahí la necesidad de replantear el proceso de forma significativa (Woo et al., 2007). En un estudio que realizó la Universidad Central de Florida (Hartman, 2005) con 1486 estudiantes on-line constató que lo que más valoraban de su proceso de formación era el proceso de comunicación y la sensación de pertenencia a una comunidad. Respecto a los formadores lo que más valoraron fue:

- Facilitar a los alumnos su proceso de aprendizaje

- Comunicar ideas e información de manera efectiva

- Demostrar un interés "real” por el proceso de aprendizaje del estudiante

- Organizar el proceso de formación de manera efectiva

- Asesorar el proceso de aprendizaje de los estudiantes de manera efectiva.

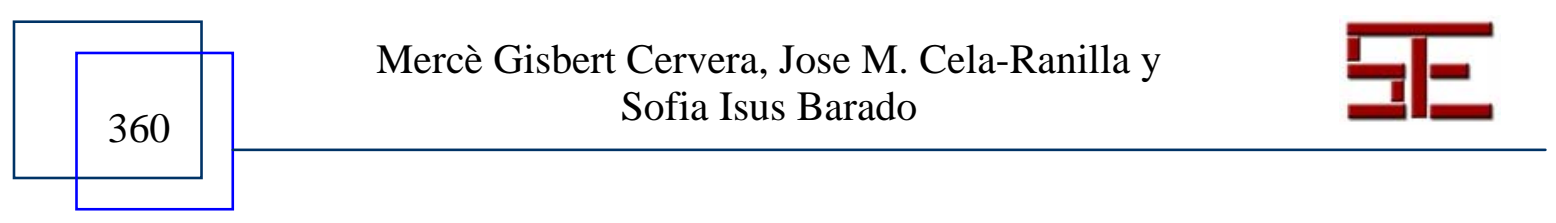




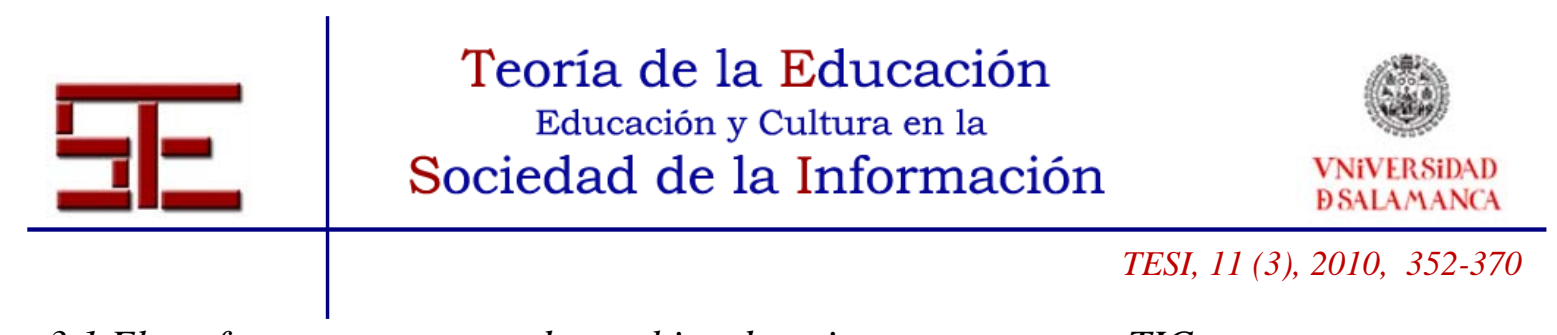

\subsection{El profesor como agente de cambio educativo en un entorno TIC}

Las TIC favorecen claramente la idea de que lo verdaderamente importante es la consecución de unos objetivos y un grado óptimo de calidad más que la presencia física en un lugar y tiempo concretos a la vez que permite generar espacios virtuales compartidos (de relación, de investigación, de trabajo). La idea de la globalización y de la internacionalización cada vez se extiende con más fuerza por lo que será fundamental que los profesores sean capaces de desarrollar proyectos transnacionales basados en:

- Trabajo interdisciplinar

- Utilización de la informática como herramienta de trabajo.

- La red como canal de comunicación.

- Las redes como espacio cooperativo y de formación.

- $\quad$ Las redes como espacio de trabajo.

Las funciones del docente cambian cuando debe desarrollar sus actividades en un entorno virtual de Enseñanza-Aprendizaje (E-A) que además deja de tener limitaciones geográficas, físicas, temporales y que tiende a dar respuesta a grupos de alumnos cada vez más heterogéneos y diversos (en el sentido más extenso de estas dos palabras), y por ello creemos que podemos realizar una serie de precisiones a su tarea.

En primer lugar, hemos de tener en cuenta el hecho de que el docente tenga una actitud positiva o negativa a la hora de desarrollar su tarea en entornos tecnológicos estará fuertemente condicionada por:

- $\quad$ La infraestructura de comunicaciones de que disponga.

- $\quad$ El espacio disponible en su centro habitual de trabajo que permita la fácil integración de la tecnología.

- Su preparación para el uso de esta tecnología (tanto desde el punto de vista del hardware como del software).

- La disponibilidad del docente para una formación permanente que la garantice no perder la "carrera tecnológica”.

En segundo lugar, debe ser capaz de cambiar sus estrategias de comunicación, pues es distinto hablar a un auditorio presencial que hacerlo a un auditorio virtual. La comunicación verbal dependerá de la calidad de las comunicaciones, en muchas ocasiones más que de la fluidez del orador. En cuanto a la comunicación no verbal, y aún en el caso de poder transmitir imagen a tiempo real, esta carece de mucho sentido.

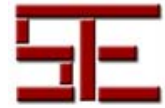

Mercè Gisbert Cervera, Jose M. Cela-Ranilla y Sofia Isus Barado 


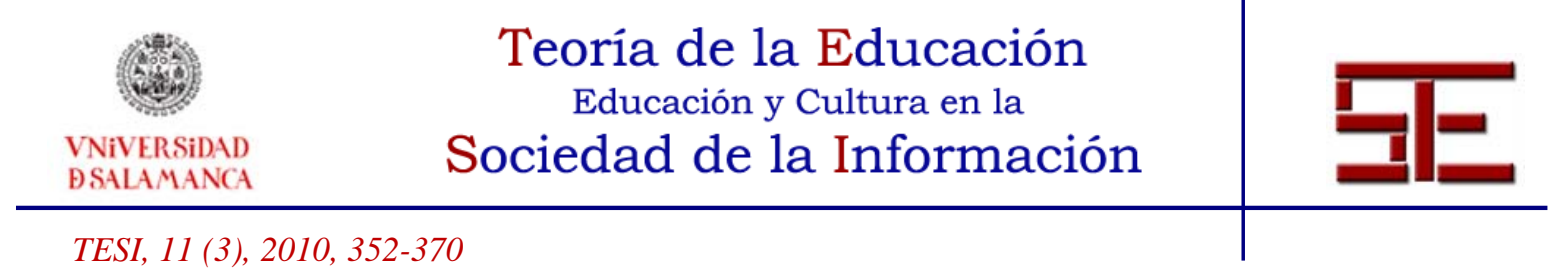

En tercer lugar, el docente debe estar preparado para hablar delante de una cámara, y delante de una cámara y unos alumnos presenciales si la sesión se diseña para alumnos presenciales y alumnos virtuales.

Los ejes espacio-temporales y los espacios tangibles que han constituido, hasta ahora, los elementos fundamentales en la organización de los procesos educativos cambian totalmente de sentido. El tiempo es relativo y el espacio intangible, por tanto, el profesor deberá buscar otros referentes para planificar y gestionar su tarea, tanto docente como organizadora y de gestión.

Todos estos condicionantes hacen necesario el establecimiento de unos programas orientados a la capacitación del profesorado. Una formación cuyo contenido no se reduzca al de usuario de la tecnología sino que le permita dar un salto cualitativo en sus diferentes propuestas didácticas.

En este sentido, aunque todas las administraciones han invertido muchos recursos desde la década de los 80 del siglo XX, en la capacitación del profesorado en TIC y en la dotación de los centros educativos, aún persisten algunos problemas que deben tenerse presentes a la hora de revisar y rediseñar políticas TIC por parte de éstas administraciones. Los principales problemas que aún persisten y que debemos tener en cuenta son:

- Un problema lingüístico derivado del poco dominio de la lengua inglesa, lengua mayoritaria y dominante en la red (no hemos de olvidar, de todos modos, que la mayor parte de población de la red es de habla inglesa).

- Una insuficiente oferta informativa y formativa en nuestra red en lengua española.

- Un problema lingüístico derivado del poco dominio de la lengua inglesa, lengua mayoritaria y dominante en la red (no hemos de olvidar, de todos modos, que la mayor parte de población de la red es de habla inglesa)

Por su parte, el sistema educativo debería:

- Favorecer el aprendizaje y el dominio de las nuevas herramientas de comunicación fundamentales para garantizar la competitividad de los alumnos.

- Diversificar el formato de los materiales pedagógicos y tender hacia la utilización de materiales didácticos en formato electrónico.

- Utilizar el ordenador como herramienta, medio y recurso habitual favorecedor de los procesos de aprendizaje y de acceso a la información, la cultura y la formación.

- Utilizar el ordenador como herramienta, medio y recurso habitual favorecedor de los procesos de aprendizaje y de acceso a la información, la cultura y la formación.

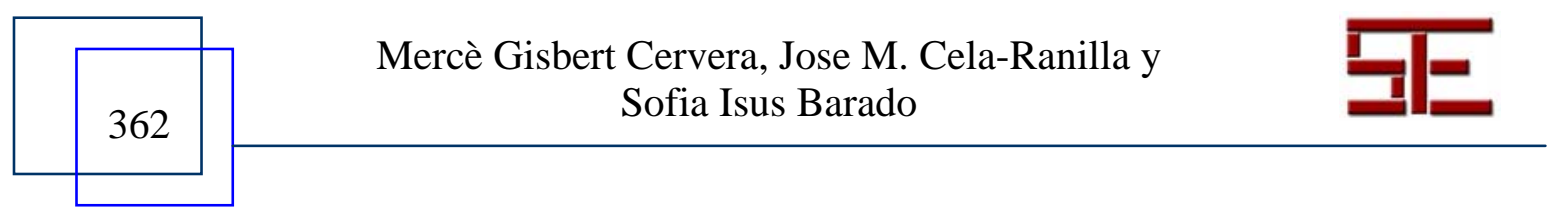




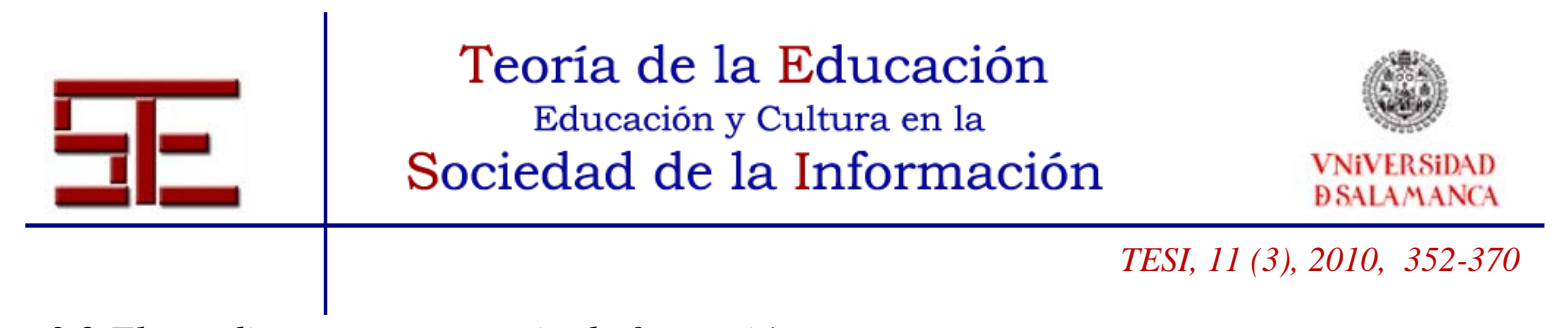

\subsection{El estudiante como usuario de formación}

El otro gran agente educativo, el estudiante, tiene ante sí actualmente una posibilidad que constituye un auténtico reto: Aprender por uno mismo, autoaprender. Considerando el impacto de las TIC en los procesos formativos y la continuidad de éstos, todo parece indicar que una modalidad de formación que imperará en el tercer milenio será la autoformación y un aspecto de ésta, el autoaprendizaje.

De alguna manera, esta realidad tecnológica será una plataforma ideal para consolidar ese modelo de educación centrado en el alumno que, como se decía anteriormente, es una de las claves del nuevo espacio universitario europea. Este modelo se concreta en una concepción activa y una secuencia educativa orientada al aprendizaje activo.

Hasta ahora eran las instituciones educativas formales las que tenían el peso fundamental de la educación y la formación y a las que se pedía explicaciones de los buenos o malos resultados del nivel de formación de una comunidad y así continua siendo. Cada vez más, los individuos aprenden más cosas fuera de las instituciones educativas que en ellas, en esto han tenido mucho que ver las TIC.

Las TIC han generado toda una serie de procesos de transmisión de la información y del conocimiento paralelo, complementario e incluso alternativo a la institución escolar a los de la escuela. ¿Podemos decir mejores? No diríamos tanto pero la verdad es que presentados de un modo más flexible, más motivador y más próximos al usuario (en este caso los estudiantes).

En este momento en que las instituciones escolares, hasta hace pocos años transmisoras de información y de conocimiento por excelencia, se ven superadas, es imprescindible revisar sus funciones en el sentido que los estudiantes que a ellas acuden no necesitan tanta información como herramientas para poder ordenarla, sistematizarla, comprenderla y utilizarla de manera crítica.

Avanzando en esta idea de formación, no resulta difícil imaginar unos contenidos y objetivos de la formación más centrados en aspectos como el autoempleo, la ocupación, el desarrollo personal o el desarrollo comunitario. En esta nueva realidad no será suficiente con conocer y "controlar" nuestro espacio vital actual (la familia, la empresa para la que trabajamos y el pueblo o ciudad en el que vivimos) sino que los horizontes de trabajo y de relación se ampliarán tanto que será imprescindible asumir y entender aquellas realidades y situaciones que hasta ahora nos parecían muy lejanas. Será muy importante tener capacidad para entender y favorecer el desarrollo comunitario desde un punto de vista amplio.

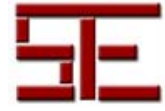

Mercè Gisbert Cervera, Jose M. Cela-Ranilla y Sofia Isus Barado 


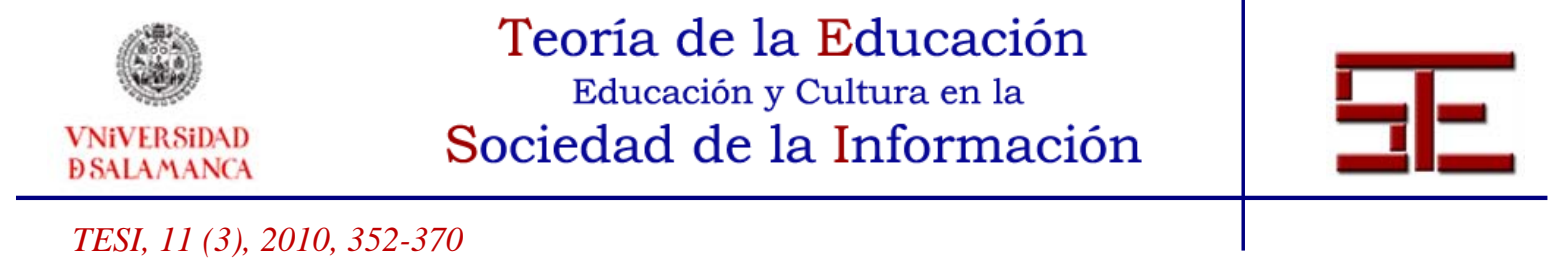

\section{4.- LOS ENTORNOS TECNOLÓGICOS DE SIMULACIÓN EN LA UNIVERSIDAD: APRENDER HACIENDO}

Es fundamental tener en cuenta que la mayor parte de dispositivos tecnológicos que utilizan los estudiantes desde muy pequeños tienen más que ver con un proceso activo de interacción que con procesos de lectura pasiva. Del mismo modo, todos ellos se han alfabetizado tecnológicamente a partir de la imagen [principalmente la televisión]. El informe de EDUCAUSE (2006) aborda de forma predictiva esta temática identificando seis áreas a considerar:

- Social computing: Aplicaciones del ordenador encaminadas a facilitar la interacción y la colaboración.

- Personal brodcasting basados en el audio y el video. Esto ha sido posible gracias a la simplificación de las herramientas para el tratamiento del audio y video y a la mejora de las infraestructuras tecnológicas.

- Telefonía móvil aproximando los contenidos y los servicios educativos a los usuarios.

- Juegos educativos con un gran potencial formativo.

- Realidad Aumentada, comúnmente utilizada en áreas como medicina, ingeniería, arqueología, etc. Su mayor aportación es la creación de realidades y espacios 3D a partir de datos abstractos que permiten reproducir con exactitud espacios reales pero en un formato digital.

- Context-Aware, entornos y dispositivos desarrollados para responder a la voz, los movimientos o cualquier otro tipo de señales sutiles realizadas por los ocupantes de un contexto.

Las investigaciones sobre el uso del ordenador evidencian que entre los 5 y los 8 años un $20 \%$ de la población ya usa el ordenador y entre los 16 y los 18 años se convierte en una herramienta de uso diario (Oblinger, 2005; KFF, 2003; Grunwald, 2004). Esto indica que todos los estudiantes que llegan a la universidad llegan alfabetizados tecnológicamente. No sólo son las herramientas informáticas las que constituyen el contexto habitual de los estudiantes sino, como ya hemos mencionado, toda una serie de dispositivos tecnológicos (móviles, TV, videojuegos,...) que hacen de esta generación la generación .net. (Oblinger, 2005; McNeely, 2005).

Toda esta tecnología no sólo está presente en los espacios formativos sino que forma parte también de la vida cotidiana. Esta integración de la tecnología para la vida diaria (lo que se ha denominado en las últimas investigaciones e-Live (Windham, 2005) para la toma de decisiones, para la comunicación, para el acceso a la información, permite que los jóvenes se sientan cómodos en un entorno tecnológico. Es de ello de lo que tendremos que valernos en la universidad para generar otros espacios para el

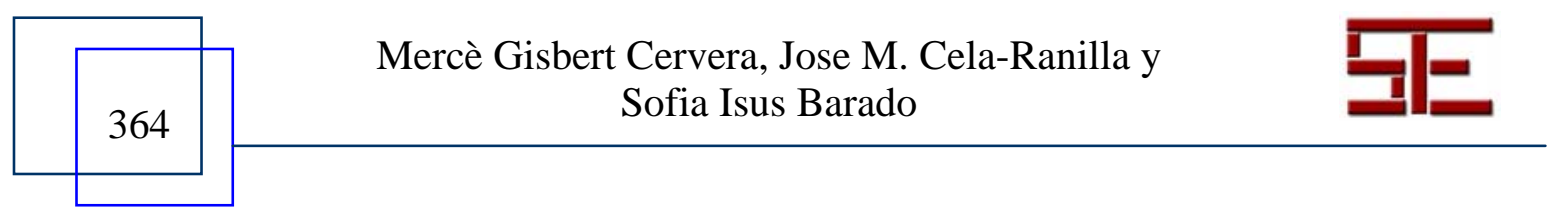




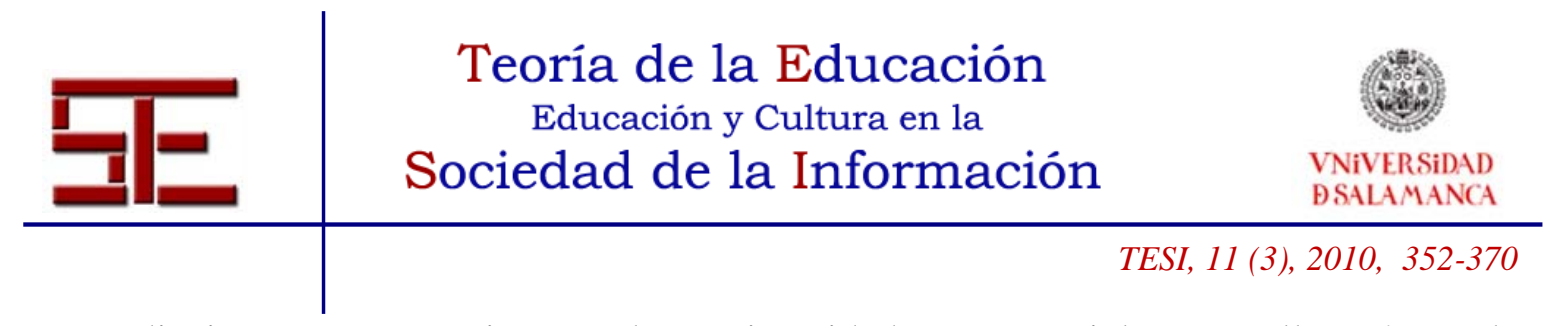

aprendizaje. Estos espacios en las universidades presenciales nos llevarán a la configuración de proyectos de Blended-Learning para poder rentabilizar nuestra experiencia en procesos presenciales al tiempo que aprovechar la potencialidad de las TIC (Dziuban et al., 2004).

En un estudio realizado en 3 universidades de USA con 4363 estudiantes éstos manifestaban: un 41,2\%, que prefería un uso moderado de la tecnología y en 30,8\% un uso intensivo; sólo un 2,2\% manifestó preferir estudios on-line. En la misma investigación se demostró que los estudiantes de grado preferían entornos con menos tecnología mientras que los estudiantes de máster (en casi un 70\%) preferían procesos de formación con TIC. Por áreas de conocimiento los estudiantes que más preferían entornos TIC eran los de Ingeniería y Economía y Negocios, frente a los de arte, que eran los más reacios a utilizarlos. Los estudiantes de educación se situaban en un nivel intermedio (Kvavik, 2004, 2005).

\section{1- El valor formativo de las simulaciones.}

Desde hace una década, más o menos desde que se produce la explosión de Internet en Europa, se evidencia el final de los entornos de formación exclusivamente cara a cara. Cada vez es más evidente que los procesos de aprendizaje realmente efectivos son aquellos que implican probar, construir, experimentar, tomar decisiones, resolver problemas,... en definitiva, todos aquellos procesos que requieren una posición activa del estudiante. También son estas situaciones de aprendizaje las que le permiten al estudiante adquirir competencias y generar conocimiento con más facilidad.

Siguiendo a Millán (1997), las simulaciones, en cualquiera de sus modalidades (estudio de casos, role playing, juegos, etc) pueden encontrar una secuencia estructural identificando sus componentes como elementos constitutivos a analizar y utilizar en términos educativos. Una simulación podría ser descrita como una representación de una realidad observada o imaginada que obliga a tomar decisiones de acción que tienen efectos con su correspondiente retroalimentación.

Esta descripción analítica de las simulaciones permite manipular sus elementos orientando y definiendo su diseño con una intencionalidad educativa concreta definida por el formador.

Por su similitud a los entornos laborales y su versatilidad, las simulaciones se convierten en una metodología muy valiosa en el aprendizaje de competencias transversales considerando sus características como entorno formativo así como la percepción que el estudiante tiene de su uso.

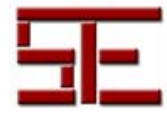

Mercè Gisbert Cervera, Jose M. Cela-Ranilla y Sofia Isus Barado 


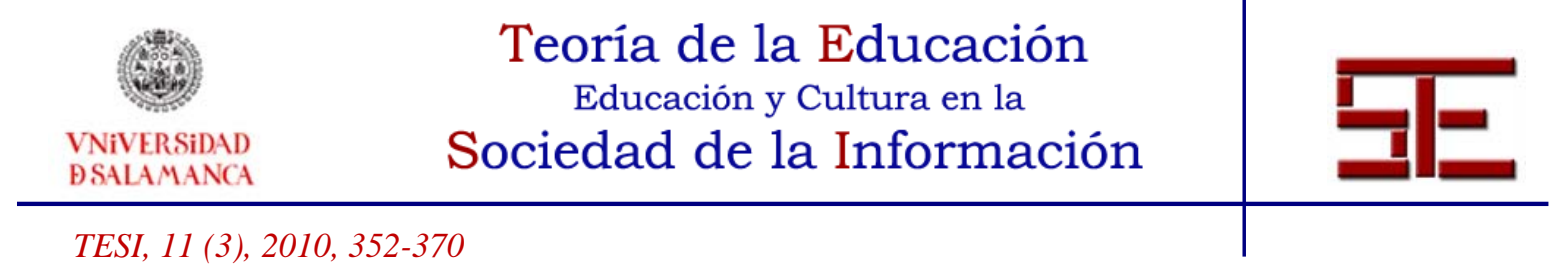

Las aportaciones clave de los entornos basados en simulaciones son:

- Requieren la ejecución de tareas individuales

- Tienen un alto valor los resultados de las acciones que se van desarrollando para la solución última del caso, situación o problema.

- Resultan un instrumento válido para la evaluación y la demostración de la adquisición de competencias.

- Simulan espacios de trabajo reales que reflejan problemas del mundo real.

- Transforman la preparación en experiencia a través de una evaluación continua de las competencias que se están trabajando.

En cuanto a la percepción que el estudiante tiene del uso de las simulaciones en entornos virtuales los estudiantes pueden:

- Observar y/o acceder a datos e información de sitios remotos, entornos o procesos.

- Relacionar datos visibles e invisibles.

- Facilitar la manipulación de entornos y de variables.

- Influir sobre cambios y/o procesos.

- Practicar competencias que sería complicado desarrollar en la vida real.

Las exigencias del actual mundo laboral y profesional favorecen la creación de espacios de formación que cumplan con la misión de preparar para la solución de problemas reales en tiempo real.

\section{5.- A MODO DE CONCLUSIÓN}

El sentido global del artículo trata de conciliar tres conceptos que son realidades de facto en nuestros días: la emergencia de un nuevo perfil de estudiante universitario, un cambio en el sistema de requerimientos desde el mundo laboral en términos de competencias, más específicamente las competencias transversales, y la necesidad de explorar estrategias de formación basadas en una tecnología compleja pero disponible como son las simulaciones en entornos digitales.

A partir de este conjunto de reflexiones se pueden apuntar líneas de investigación proponiendo algunas preguntas que a día de hoy aparecen como no resueltas:

- ¿ ¿Cuáles son las mejores situaciones de aprendizaje en que pueden ser usadas las simulaciones en entornos TIC?

- ¿ ¿ $\quad$ Son más efectivas las simulaciones en procesos de autoaprendizaje o en procesos en proyectos blended o presenciales?

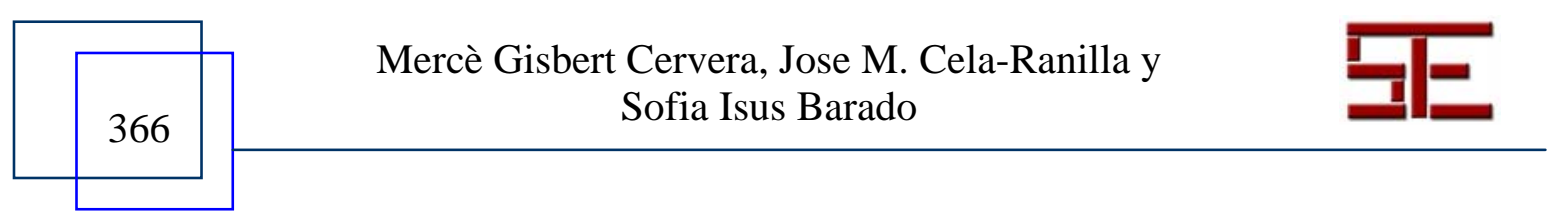




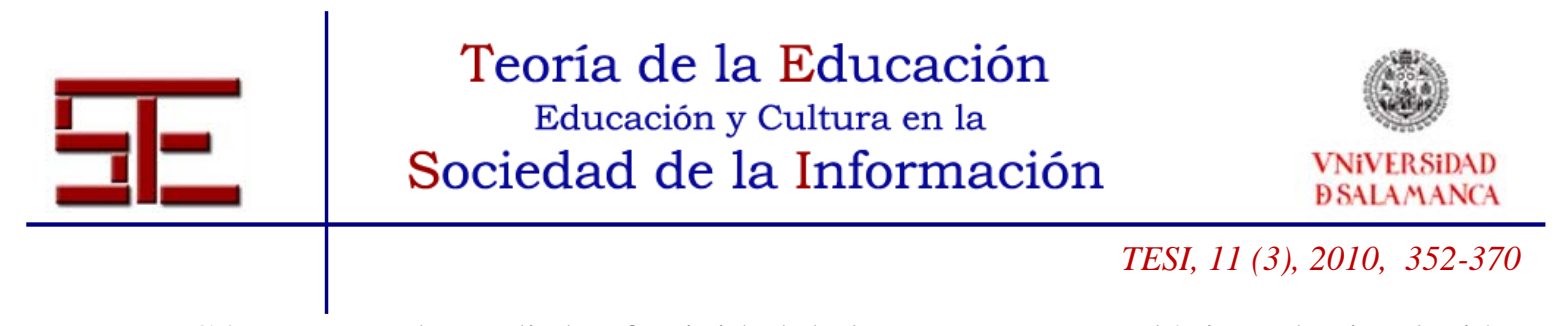

- ¿ ¿Cómo se puede medir la efectividad de los entornos tecnológicos de simulación para la adquisición de competencias?

- ¿ ¿Son más efectivos los procesos de simulación a través de

imágenes/animaciones o a través de procesos de experimentación a partir de la manipulación?

- ¿ ¿Cuál debe ser el rol del profesor en un proceso de formación en entornos de simulación? ¿Qué formación requiere para ello?

- ¿ ¿Cuál es el equilibrio óptimo entre los presencial y lo virtual en un proyecto formativo de estas características?

Alguna de las respuestas a estas preguntas pretenden ser encontradas con la ejecución del proyecto SIMUL@ (SIMUL@: Evaluación de un Entorno Tecnológico de Simulación para el Aprendizaje de Competencias Transversales en la Universidad Ref. EDU2008-01479) desarrollado conjuntamente por la Universidad de Lleida, la Universidad de Hamburgo, la Universidad do Minho y que coordina la Universidad Rovira y Virgili.

\section{6.- BIBLIOGRAFÍA.}

Bunk, G.P. (1994). La transmisión de las competencias en la formación y perfeccionamiento profesionales de la R.F.A. En: Revista Europea de Formación Profesional, 2, (8-14). Berlín: CEDEFOP.

Claxton, G. (2007). Expanding young people's capacity to learn. British Journal of Educational Studies, (vol. 55) (2).

Connolly, T., Stansfield, M.,From e-learning to games-based e-learning: using interactive technologies in teaching an IS course (vol. 6), Number 2-4 / 2007 ,188 - 208.

De Miguel, M (2005). Modalidades de enseñanza centradas en el desarrollo de Competencias. Universidad de Oviedo.

Dziuban, C. D. et al. (2004). Blended Learning. Boulder, Colo. EDUCAUSE Center for Applied Learning, reserarch bulletin, issue 7.

EDUCAUSE (2005). The Horizon 2006 Report. The New Media Consortium and EDUCAUSE Learning

Initiative. [http://www.educause.edu/ir/library/pdf/CSD4387.pdf]

Ezziane, Z. (2007). Information Technology Literacy: Implications on Teaching and Learning. Educational Technology \&

Fink, I. (2002). Classroom Use and Utilization. Facilities Manager, 19. no. 2 (mayjune).

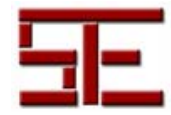

Mercè Gisbert Cervera, Jose M. Cela-Ranilla y

Sofia Isus Barado 


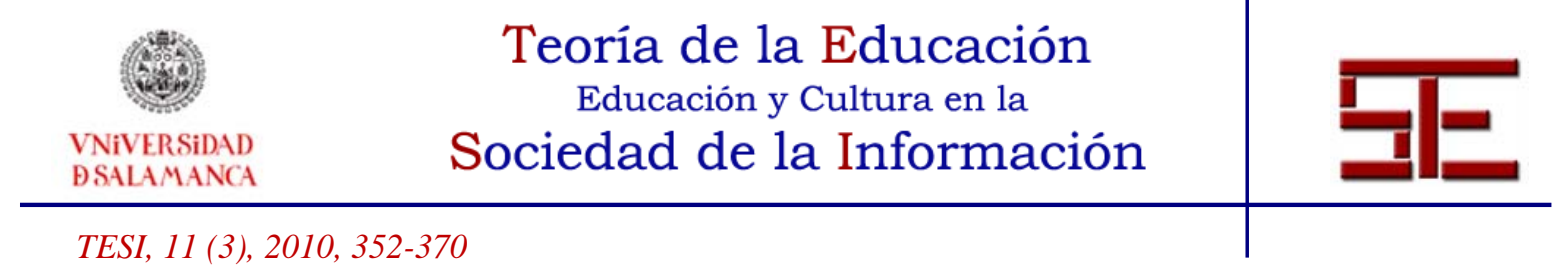

Ginns, P., Ellis, R. (2007). Quality in blended learning: Exploring the relationships between on-line and face-to-face teaching and learning. Internet and Higher Education 10 (2007) 53-64.

Grunwald, P. (2004). Children, Families and the Internet. Bethesda. Md. Grunwald Associates. http://www.grunwuald.com.

Hartman, J. et al. (2005). Preparing the Academy of Today for the Learning of Tomorrow. OBLINGER, D. \& OBLINGER, J.: Educating the net Generation. EDUCAUSE.

Kaiser Family Foundation (2003). New Study Finds Children Age zero to Six Spend as Much time with TV, Computers and Video games playing out side. [http://www.kff.org/entmedia].

Kvavik, R. (2005). Convivence, Communications and Control: How Students Use Technology. OBLINGER, D. \& OBLINGER, J.: Educating the net Generation. EDUCAUSE.

Kvavik, R. B. et al. (2004). ECAR Study of Students and IT, 2004 : Convivence, Research and Control. EDUCAUSE. Research Study. vol. 5.

Lucas Mangas, S. (2007). Desarrollo de competencias desde la Enseñanza Universitaria. Armonización con la Educación Secundaria y el mercado de trabajo, desde la Psicología Social de la Educación. Electronic Journal of Research in Educational Psychology vol. 5 (1): pp: 125-158

McNeely, B. (2005). Using Technology as a learning tool. No Just the Cool New Thing. Oblinger, D. \& Oblinger, J.: Educating the net Generation. EDUCAUSE.

MEC (2006). Propuestas para la renovación de las metodologías educativas en la Universidad. Ministerio de Educación y Ciencia.

Michavila, F. (2004). Las innovaciones educativas basadas en las TIC en la formación universitaria presencial y a distancia. Programa de estudios y análisis de la Dirección general de Universidades. MEC.

Mitchell, W. J. (2004). Rethinking Campus a Classroom Designe. NIII. Fall Focus Session Cambridge Mass.

Millán, M.D. (1997). La simulación y la representación de la realidad. En Estrategias de simulación: Ora, un modelo innovador para aprender del medio. De la Torre, S. (coord.) Ed. Octaedro.

Mora, J. G. (2005). Análisis y diseño de estrategias para el desarrollo del aprendizaje permanente en Europa. El caso de la educación continua universitaria en el EEES y la definición de indicadores de desarrollo de la vinculación universitaria

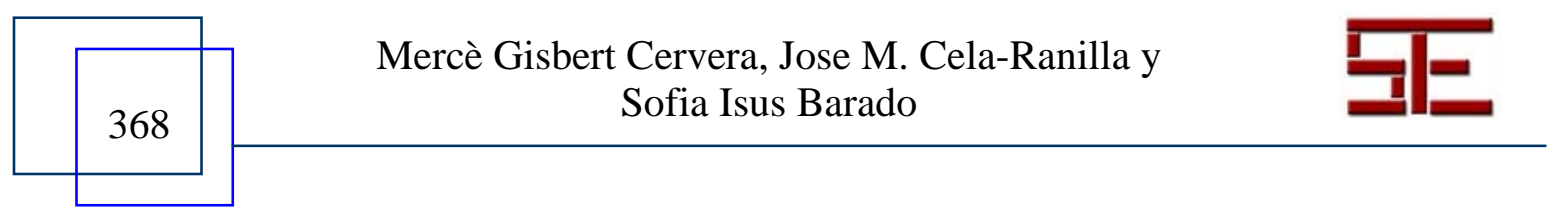




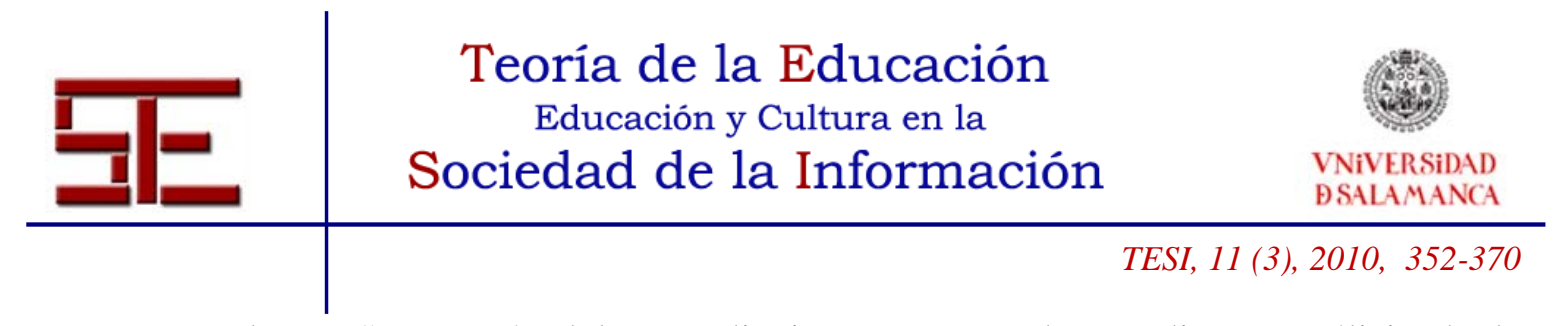

en el EEES a través del aprendizaje. Programa de estudios y análisis de la Dirección general de Universidades. MEC

Nelson, P. B. et al. (2000). Acoustical Barriers to Learning: Children at Risk in Every Classroom Language, Speech and Hearing Services in Schools, 31. Pp. 356-61.

NLII Y EDUCAUSE (2004). Learning Transition from Classrooms to Learning Spaces. NLII White Paper.

Oblinger, D. (2004). The Next Generation of Educational Engagement. EDUCAUSE. [http://jime.open.ac.uk/2004/8/]

Oblinger, D. (2006). Simulations, Games and Learning. EDUCAUSE Learning Initiative.

Oblinger, D. y Oblinger, O. (Eds).(2005). Educating the Net Generation. EDUCAUSE.

Pagani, R. (2002). El crédito europeo y el sistema educativo español. Informe tecnico. Madrid PRADES, A. (2005). Les competències transversals i la formació universitària. Tesis Doctoral. UB. Consulta en línia: http://www.tdcat.cesca.es/ .

Reaessens, J. \& Goldstein, J. H. (2005). Handbook of computer game studies. Cambridge, Mass. [u.a.]: MIT Press.

Roberts, G. et al. (2005). Technology and Learning Expectations of the net generations. Oblinger, D. \& Oblinger, J.: Educating the net Generation. EDUCAUSE.

Rodriguez Moreno, M. L. (2006). De la Evaluación a la Formación de Competencias Genéricas: Aproximación a un Modelo. Revista Brasileira de Orientação Profissional vol. 7 (2): pp. 33-48

Scott-Weber, L. (2004). In Sync: Environmental Behavior Reserarch and the Desing of Learning Spaces. Ann Arbor. Mich. Society for Collage and University Planning.

Squire, K. (2002). Cultural Framing of Computer/Video Games. The international journal of computer game research , 2(1).

Tondeur, J.,van Braak, J,; Valcke, Martin. (2007). Curricula and the use of ICT in education:Two worlds apart? British Journal of Educational Technology Vol 38 No 6. 962-976.

Valenti, M. (2002). Creating the classroom of the future. EDUCAUSE Review. September/October.

White, S. (2007). Critical success factors for e-learning and institutional change-some organisational perspectives on campus-wide e-learning. British Journal of Educational Technology. vol $38 \mathrm{~N}^{\circ} 5$.

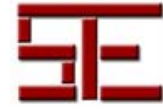

Mercè Gisbert Cervera, Jose M. Cela-Ranilla y Sofia Isus Barado 


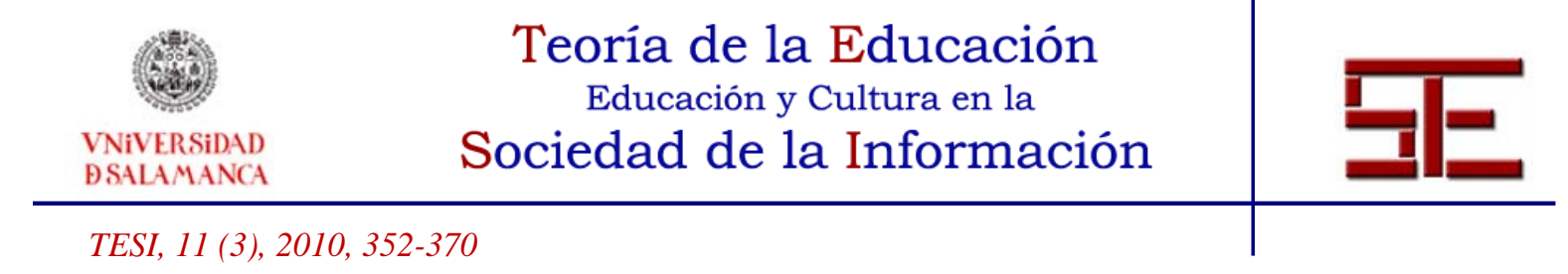

Whitehouse, K. (2005). Web-Enabled Simulations: Exploring the Learning Process. EDUCAUSE Quarterly. $N^{\circ} 3$.

Windham, C. (2005). The Students Perspective. Oblinger, D. \& Oblinger, J.: Educating the net Generation. EDUCAUSE.

Woo, Younghee; Reeves, Thomas C. (2007). Meaningful interaction in web-based learning: A social constructivist interpretation. Internet and Higher Education 10. $15-25$

Xu, Yonghong (Jade), Meyer, Katrina A. (2007). Factors explaining faculty technology use and productivity. Internet and Higher Education 10. 41-52

Para citar el presente artículo puede utilizar la siguiente referencia:

Gisbert Cervera, M., Cela-Ranilla, J. y Isus Barado, S.: (2010). "Las simulaciones en entornos TIC como herramienta para la formación en competencias transversales de los estudiantes universitarios”. En De Pablos Pons, J. (Coord.) Buenas prácticas de enseñanza con TIC [monográfico en línea]. Revista Electrónica Teoría de la Educación: Educación y Cultura en la Sociedad de la Información. Vol. 11, n ${ }^{0} 1$. Universidad de Salamanca, pp. 352-370. [Fecha de consulta: dd/mm/aaaa]. http://revistatesi.usal.es/ revistas_trabajo/index.php/revistatesi/article/view/6309/6322 ISSN: 1138-9737

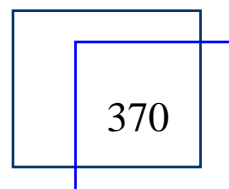

Mercè Gisbert Cervera, Jose M. Cela-Ranilla y Sofia Isus Barado 